\title{
Angle-closure glaucoma: diagnosis
}

\author{
Glaucoma de ÂNGULO FECHADO: DIAGNóstico \\ Authorship: Brazilian Council of Ophthalmology \\ Final preparation: September $19^{\text {th }}, 2013$ \\ Participants: Jair Giampani Jr, Ricardo Simões, Wanderley Marques Bernardo
}

The Guidelines Project, an initiative of the Brazilian Medical Association, aims to combine information from the medical field in order to standardize procedures to assist the reasoning and decision-making of doctors.

The information provided through this project must be assessed and criticized by the physician responsible for the conduct that will be adopted, depending on the conditions and the clinical status of each patient.

\section{Description OF the eVIDENCE COLLECTION METHOD:}

The literature review of scientific articles in this guideline was held in the databases Medline, Cochrane and SciELO. The search for evidence came from actual clinical scenarios and used keywords (MeSH terms) grouped in the following syntax: (glaucoma, angle-closure or glaucoma, closed angle or glaucomas, closed-angle or glaucoma, uncompensative or glaucomas, uncompensative or angle closure glaucoma or angle closure glaucomas or glaucomas, angle closure or glaucoma, narrow-angle) and (gonioscopy or tomography optical coherence or microscopy, acoustic or dark adaptation). The articles were selected after critical evaluation of the strength of scientific evidence, and publications of greatest strength were used for recommendation. The recommendations were drawn from group discussion. The entire guideline was reviewed by an independent group specializing in evidence-based clinical guidelines.

\section{Degree OF RECOMMENDATION AND STRENGTH OF EVIDENCE:}

A: Experimental or observational studies of higher consistency.

B: Experimental or observational studies of lower consistency.

C: Case reports (non-controlled studies).

D: Opinions without critical evaluation, based on consensus, physiological studies, or animal models.

Objective: To assess the main diagnostic methods used in angle-closure glaucoma in the light of the best available evidence.

Conflict of interest: No conflict of interest informed.

\section{INTRODUCTION}

Glaucoma is the generic name of an optic neuropathy with multifactorial etiology characterized by progressive damage to the optic nerve, with consequent impact on the visual field. Even though it can present with intraocular pressures considered within the normal range (normal tension glaucoma), elevation of intraocular pressure (IOP) is the main risk factor. The most common type is the primary open angle glaucoma, often asymptomatic. Another type, called primary angle-closure glaucoma, is characterized by occlusion of the anterior chamber angle due to anatomic conditions that produce overlapping or adhesion of the peripheral iris to the outer surface of the camerular sinus, with consequent elevation of intraocular pressure, damage to the optic disc and/or corresponding visual field defect ${ }^{1.2}(\mathbf{D})$. Acute primary angle closure (formerly called primary acute glaucoma) can cause severe eye pain, headache, nausea, vomiting, elevated IOP (often above $40 \mathrm{~mm} \mathrm{Hg}$ ) and reduced visual acuity, being considered an ophthalmic emergency and requiring immediate treatment ${ }^{2}(\mathbf{D})$.

While glaucoma may or may not be accompanied by various symptoms, an almost inevitable complication is irreversible visual loss, affecting first the peripheral vision. Earlier loss is subtle and may go unnoticed by the patient. In the late stages of the disease, moderate to severe losses occur with impairment of central vision, sometimes progressing to blindness.

\section{Can ultrasound biomicroscopy (Ubm) REPLACE GONIOSCOPY IN THE DIAGNOSIS OF PATIENTS WITH ANGLE-CLOSURE GLAUCOMA?}

Gonioscopy consists in the biomicroscopic exam of the topography of the anterior chamber angle (camerular si- 
nus), where the aqueous fluid has access to drainage pathways. This is the most important exam for the classification of glaucoma, and it is used as a reference for the evaluation of the anterior chamber angle configuration. The identification of all anatomical details of the camerular sinus allows the assessment of crucial aspects for diagnosis of several types of glaucoma ${ }^{3}(\mathbf{D})$. Performed using lenses (direct gonioscopy) or by using the image reflected on a mirror attached to the lens (indirect gonioscopy with 1-4 mirror lens), it allows to establish whether a particular angle is open or closed, and if closed, to what extent ${ }^{4}(\mathbf{D})$. It also allows, through maneuvering (indentation), differentiation between the overlapping of the iris and the outer surface of the camerular sinus, and true goniosynechia. Nevertheless, the findings of gonioscopy may be compromised by excessive pressure on the lens or intensity of lighting, which tends to increase the opening angle of the anterior chamber.

UBM, a diagnostic method described in the early 1990s, uses a high frequency transducer $(50-100 \mathrm{MHz})$, thus permitting an axial and lateral resolution of around 20 to 40 micra, even though at the expense of a reduction in ultrasound penetration (approximately 5 millimeters) ${ }^{5}$ (C). The theoretical advantages of the method are the possibility of evaluating retro iridian structures and performing quantitative measurements of the camerular sinus. Its main limitations are: high cost, the dependence of a qualified examiner, the observation of a restricted region of the camerular sinus, and the need for dipping the ultrasound $\operatorname{probe}^{4}(\mathbf{D})$.

In a study involving Chinese subjects with suspected primary angle closure (characterized as the inability of seeing through gonioscopy the pigmented portion of the trabecular meshwork at 180 degrees or more), the overlapping of the iris and the outer surface of the camerular sinus was most commonly detected by UBM than gonioscopy. The prevalence of overlapping found using UBM totaled $15.4 \%$ in patients with angle grade 4 Shaffer, $45 \%$ in those with grade $3,71 \%$ in grade $2,70.2 \%$ in grade 1 , and $86.4 \%$ in grade $0^{6}(\mathbf{B})$.

Despite published papers on the use of UBM, the literature lacks studies with adequate design and sample, comparing the use of gonioscopy and UBM for the diagnosis of angle-closure glaucoma ${ }^{7-10}(\mathbf{B})^{4}(\mathbf{D})$.

\section{Recommendation}

UBM proved to be a useful method for quantitative evaluation of camerular sinus and its structures and may complement, but not replace, the semi-quantitative analysis done by gonioscopy.

\section{Can anterior segment optical coherence TOMOGRAPHY (AS-OCT) REPLACE GONIOSCOPY IN THE DIAGNOSIS OF PATIENTS WITH ANGLE- CLOSURE GLAUCOMA?}

The assessment of the dimensions and configuration of the anterior chamber angle makes up an essential part of the diagnosis and monitoring of patients with closed angle. As previously mentioned, indirect gonioscopy has been used as a traditional method and reference test for the diagnosis of primary angle-closure glaucoma. However, this method has limitations, which are mainly dependent on the examiner's experience ${ }^{11}(\mathbf{C})$. AS-OCT operates by a mechanism similar to ultrasound but uses a beam of light instead of sound waves to study the depth of tissues $^{12}(\mathbf{D})$. The initial description of this non-invasive and non-contact method applied in the study of ocular structures was made in 1991 and, since then, several studies have reported its utility in the evaluation of anterior and posterior segments of the eye $\mathrm{e}^{7,13-15}(\mathbf{B})^{16}(\mathbf{D})$. AS-OCT allows for the documentation and evaluation of the profile of the iris and its relations with other anatomical structures of the anterior segment ${ }^{17}(\mathbf{D})$. Its main limitations are due to the impossibility of assessing retro iridian structures and the high costs.

Furthermore, comparisons between the efficiency of gonioscopy and anterior segment tomography in the study of sinus camerular studies are rare. Analyzing individuals with a mean age of 62.5 years, mostly Asians (85.7\% Chinese), with suspected angle closure or confirmed primary angle closure (some of whom had been treated by iridotomy), who underwent OCT and gonioscopy, there is greater sensitivity of OCT (98\%) in the detection of closed angles ( $\geq 1$ quadrant in one or both eyes) compared with gonioscopy $(68 \%)^{18}(\mathbf{B})$. Regarding specificity, the values observed for gonioscopy (96\%) were higher compared with OCT (55\%), with a positive likelihood ratio of 1.8 and 17 for OCT and gonioscopy, respectively ${ }^{18}(\mathbf{B})$.

On the other hand, a study analyzing subjects with a mean age of 61 years ( $\mathrm{SD}=7.6$ years) mostly Asians $(92 \%$ Chinese), and without ophthalmic complaints (no suspected or confirmed angle closure), who underwent gonioscopy and OCT in order to detect closed angles (Scheie III or IV), identified through the analysis of the four quadrants of the right eye sensitivity and specificity of $66 \%$ and $79 \%$, respectively, in gonioscopy with likelihood ratio 
equal to 3.14. With respect to OCT, sensitivity and specificity of $46 \%$ and $89 \%$, respectively, were observed, with a positive likelihood ratio equal to 4.18 . The study also found greater concordance between the two methods in detecting closed angles in the upper quadrants (19\% (95 CI: 14 to 24 ) and 22\% (95 CI: 17 to 27) for OCT and gonioscopy, respectively ${ }^{19}(\mathbf{B})$.

The fact that there are few comparative studies, combined with the high cost of OCT makes it a complementary technology in the study of the anterior segment of the eye. Gonioscopy, despite its semi-quantitative limitations, remains the method of choice for evaluating the periphery of the anterior chamber and camerular sinus in patients with angle-closure glaucoma.

\section{Recommendation}

AS-OCT proved to be a useful method for quantitative evaluation of camerular sinus and its structures and may complement, but not replace, the semi-quantitative analysis done by gonioscopy.

\section{WHAT IS THE VALIDITY OF THE DARK-ROOM PRONE POSITION TEST FOR THE DIAGNOSIS OF ANGLE-CLOSURE GLAUCOMA?}

Provocation tests, including the dark-room test and the prone position test, account for physiological functional tests, which have the purpose of assessing the likelihood of angle closure with increased IOP. In the literature, the available data regarding the dark room test, first described in 1910, indicate the possibility of identification of "congestive glaucoma" in a variable number of patients, with positive results observed from $11 \%$ to $78 \%$ of cases $^{20-22}$ (C). On the other hand, a study analyzing the prone-position test in patients with suspected angle closure, revealed positivity around $48.6 \%$ of $\operatorname{cases}^{23}(\mathbf{C})$. Thus, in order to increase the specificity, the combination of tests has been proposed.

Cross-sectional study analyzing the dark-room prone position test applied in relatives of patients with primary angle-closure glaucoma found positivity in $70.3 \%$ of individuals considered affected who had been identified by gonioscopy (patients with any subtype of primary angle-closure glaucoma, i.e., sub acute, acute or chronic); accuracy, however, reached only $48 \%{ }^{24}(\mathbf{B})$.

\section{Recommendation}

The dark-room prone position test, if used alone, has limited validity in the diagnosis of chronic angle-closure glaucoma. Its use, combined with other diagnostic procedures, may assist in the detection of patients at risk for angle closure.

\section{References}

1. Foster PJ. The epidemiology of primary angle closure and associated glaucomatous optic neuropathy. Semin Ophthalmol 2002;05:50 PM-8.

2. $2^{\circ}$ Consenso de Glaucoma Primário de Ângulo Fechado. JPC Vasconcelos, LM Sakata, MJL Silva e VP Costa. Sociedade Brasileira de Glaucoma, 2012.

3. Bruno CA, Alward WL. Gonioscopy in primary angle closure glaucoma. Semin Ophthalmol 2002;05:59 PM-68.

4. Friedman DS, He M. Anterior chamber angle assessment techniques. Surv Ophthalmol 2008;53:250-73.

5. Pavlin CJ, Harasiewicz K, Sherar MD, Foster FS: Clinical use of ultrasound biomicroscopy. Ophthalmology 1991;98:287-95.

6. Kong X, Foster PJ, Huang Q, Zheng Y, Huang W, Cai X, He M. Appositional closure identified by ultrasound biomicroscopy in population-based primary angle-closure glaucoma suspects: The Liwan Eye Study. Invest Ophthalmol Vis Sci 2011;52:3970-5.

7. Wang D, Pekmezci M, Basham RP, He M, Seider MI, Lin SC. Comparison of different modes in optical coherence tomography and ultrasound biomicroscopy in anterior chamber angle assessment. J Glaucoma 2009; $186: 472-8$.

8. Hirasawa H, Tomidokoro A, Kunimatsu S, Mishima K, Iwase A, Tomita G, et al. Ultrasound biomicroscopy in narrow peripheral anterior chamber eyes with or without peripheral anterior synechiae. J Glaucoma 2009;18:552-6.

9. Barkana Y, Dorairaj SK, Gerber Y, Liebmann JM, Ritch R. Agreement between gonioscopy and ultrasound biomicroscopy in detecting iridotrabecular apposition. Arch Ophthalmol 2007;125:1331-5.

10. Kashiwagi K, Tsumura T, Tsukahara S. Comparison between newly developed scanning peripheral anterior chamber depth analyzer and conventional methods of evaluating anterior chamber configuration. J Glaucoma 2006;15:380-7.

11. Foster PJ, Devereux JG, Alsbirk PH, Lee PS, Uranchimeg D, Machin D, et al. Detection of gonioscopically occludable angles and primary angle closure glaucoma by estimation of limbal chamber depth in Asians: modified grading scheme. Br J Ophthalmol 2000;84:186-92.

12. Friedman DS, He M. Anterior chamber angle assessment techniques. Surv Ophthalmol 2008;53:250-73.

13. Khor WB, Sakata LM, Friedman DS, Narayanaswamy A, Lavanya R, Perera $\mathrm{SA}$, et al. Evaluation of scanning protocols for imaging the anterior chamber angle with anterior segment-optical coherence tomography. J Glaucoma 2010;19:365-8.

14. Wong HT, Lim MC, Sakata LM, Aung HT, Amerasinghe N, Friedman DS, et al. High-definition optical coherence tomography imaging of the iridocorneal angle of the eye. Arch Ophthalmol 2009;127:256-60.

15. Aptel F, Denis P. Optical coherence tomography quantitative analysis of iris volume changes after pharmacologic mydriasis. Ophthalmology 2010;117:310.

16. Huang D, Swanson EA, Lin CP, Schuman JS, Stinson WG, Chang W, et al. Optical Coherence tomography. Science 1991;254:1178-81.

17. Izatt JA, Hee MR, Swanson EA, Lin CP, Huang D, Schuman JS, et al. Micrometer-scale resolution imaging of the anterior eye in vivo with optical coherence tomography. Arch Ophthalmol 1994;112:1584-9.

18. Nolan WP, See JL, Chew PT, Freidman DS, Smith SD, Radhakrishnan S, et al. Detection of primary angle closure using anterior segment optical coherence tomography in Asian eyes. Ophthalmology 2007;114:33-9.

19. Sakata LM, Lavanya R, Friedman DS, Aung HT, Kumar RS, Foster PJ, et al. Comparison of gonioscopy and anterior segment ocular coherence tomography in detecting angle closure in different quadrants of the anterior chamber angle. Ophthalmology 2008;115:769-74.

20. Higgitt AC. The darkroom test. Br J Opthalmol 1954;38:242.

21. Leydhecker W, Niesel P. Statistische Berechnung der ghysinogischen grenzwerte bei. Klin Mbl Augenh 1954;125:458.

22. Foulds WS. Observation on the darkroom test and its mechanism. Br J Ophthalmol 1957;41:200-7. 
23. Hyams SW, Friedmann Z, Neumann E. Elevated intraocular pressure in the prone position: a new provocative test for angle closure glaucoma. Am J Ophthalmol 1968;66:661-72.
24. Sihota R, Mohan S, Dada T, Gupta V, Pandey RM, Ghate D. An evaluation of the darkroom prone provocative test in family members of primary angle closure glaucoma patients. Eye 2007;21:984-9. 